\title{
PromChoq: A Multicriteria Decision Aid Method for Actions Ranking
}

\author{
Saddek Benabied \\ University of Tebessa, \\ computer science department, \\ Tebessa, Algeria
}

\author{
Salsabile Goucem \\ University of Tebessa, \\ computer science department \\ Tebessa, Algeria
}

\author{
Hakim Bendjenna \\ University of Tebessa \\ computer science department, \\ Tebessa, Algeria
}

\begin{abstract}
Ranking available actions or alternatives with respect to multiple, often conflicting criteria is a problem of a major interest in information and engineering. Methodologies for addressing this problem have been developed from a variety of research disciplines. An investigation of existing methods shows that most existing methods suffer various drawbacks. These shortcomings include the non consideration of interactions or dependencies between criteria. To avoid it, we present in this paper, a multicriteria decision aid method for alternatives ranking called PromChoq. PromChoq is based on Promethee method, a well known multicriteria outranking method. To consider interaction between criteria, we introduce Choquet integral and $\lambda$-fuzzy measure. In order to facilitate to decision maker using PromChoq, we developed a tool which supports its main concepts. Finally, to exploit our contribution and to show its effectiveness, an empirical study of a real selection problem (teacher's selection in recruitment test) in the University of Tebessa in Algeria is presented.
\end{abstract}

\section{Keywords}

multicriteria decision analysis; ranking problem; PROMETHEE; $\lambda$-fuzzy measure; Choquet integral; candidate selection.

\section{INTRODUCTION}

The most fundamental challenge faced by managers in both public and private sectors is the making of optimal decisions on problems that are multicriteria in nature. In recent times, the giant development in computer technology coupled with advance in theory has made decision analysis an indispensable tool in both government and in business as far as the making of multicriteria decision is concerned . It is worthwhile to note that the solution of a multicriteria problem does not only depend on the fundamental data employed in the evaluation table, but also on the decision maker [10]. There exists only a compromise solution, which partly depends on the preferences of each decision maker and as a result additional information representing these preferences is required to provide the decision maker with useful decision aid.

Multicriteria Decision Aiding (MCDA) is a field which aims at giving the Decision Maker (DM) some tools in order to enable him/her to solve a complex decision problem where several points of view must be taken into account. MCDA concentrates on suggesting "compromise solutions", taking into consideration the trade-offs between criteria and the DM's preferences [13]. The most important roles in the decision making process, based on multiple criteria analysis, play the DM and analyst. The DM is a person (or a group of people), who has a great impact on the decision making process. He/she expresses preferences, evaluates the situation, considers different solutions and approves final results. An analyst is an expert involved in every stage of the decision process. He/she recognizes the decision problematic, constructs the decision model of the situation, controls the data, explains consequences of certain decisions and selects the appropriate decision aiding tools.

Alternatively, multicriteria analysis (MA) is widely used for selecting or ranking alternatives in relation to multiple criteria [14]. They are, in particular, unable to adequately handle the subjectiveness and imprecision of the decision process and often impose a high cognitive effort on the decision maker (DM).

The application of fuzzy set theory in MA provides an effective means for modeling the subjectiveness and imprecision.. However, no single approach is exempt from criticism about its overall performance and practical use in tackling real problems. The approaches based on the aggregation model of preferences may be complex and hard to use. For the approaches developed in the context of multi attribute value theory, the ranking of the fuzzy value remains a challenging issue, as it is not straightforward but when the value function is reached, the prescription is immediately deduced from the aggregation preferences process [13].

In outranking methods, we can distinguish two phases: aggregation and exploitation. The aggregation process corresponds to the operation, which transforms the marginal evaluations of separate criteria into a global outranking relation between every pair of alternatives, which is generally not transitive nor complete. The exploitation process deals with the outranking relation in order to clarify the decision through a partial or total preordering reflecting some of the irreducible indifferences and incomparability's [33].

To some extent outranking relations offer a compromise between value functions and preference relations; their power of expressiveness is far beyond the one of value functions since they are good models for such phenomena as nontransitivity and incomparability. ELECTRE III, PROMETHEE and other methods for decision aid (e.g. [15]; [33]) build and exploit an outranking relation.

The decision-making process passes by several stages, and our work is registered under the problems of the competing actions classification (arrangement). There exist several multicriteria methods of analysis of decision-making aid, and which answer the problem of classification. Among these methods: PROMETHEE and ELECTRE method are most used. These methods are based on calculation of some index as: flow Net of each action by taking of account the various criteria of classification for PROMETHEE, and concordance, no concordance index, and different thresholds for ELECTRE. 
However, the calculation of various indexes is based on the use of the balanced sum, which does not take into account the interaction between the criteria. Effectively, the most often used operator to aggregate criteria in decision making problems is the classical weighted arithmetic mean. However, in many problems, the criteria may interact and a substitute to the weighted arithmetic mean has to be adopted. For example, consider the problem of evaluating a given car based on three criteria \{fuel efficiency, luxury, price $\}$. A highly luxurious car generally comes with a higher cost. In this case, luxury and price form positive correlating criteria, and the evaluation will be an overestimate [32].

Therefore, to fill the limits of existing outranking methods and thus to help the decision maker classifying the competing actions, by taking into account several criteria and interactions between them; we propose a baptized method PromChoq based on: PROMETHEE method, the Choquet integral and the $\lambda$ - fuzzy measure. Thereafter, we will clarify our contribution stage by stage before applying it for solving a real actions ranking problem (the classification of the candidates to the university education). PromChoq can effectively calculate the overall performance of each action, construct a complete order and exploit it to recommend to the DM a ranking of the actions.

In the following sections, we begin by providing some background on multi-criteria decision analysis area (Section 2), especially Promethee method, Choquet integral and fuzzy measure. Next, we describe the proposed process steps (Section 3). We then, illustrate it using a case study (Section 4) issued from Tebessa university in Algeria. The paper closes by outlining some conclusions and further research directions (Section 5).

\section{BACKGROUND}

In order to be as far as possible self-contained, we give in this section necessary introduction and definitions for Promethee method, Choquet integral and $\lambda$-fuzzy measure.

\subsection{Promethee method: a multicriteria decision aid method}

Decision making has been defined differently by various researchers. [34] formally defines decision making as "the study of identifying and choosing alternatives based on the values and preferences of the decision makers". According to [35], decision making is "the cognitive process leading to the selection of a course of action among alternatives". It can be deduced from these two definitions that decision making involves DMs, options and selecting a final solution in a clear and transparent manner.

In real-world, most decision situations are complex demanding multiple perspectives from different point of view.

The classification of MCDA methods corresponds to the above classification of multiple criteria decision problems. Thus, one can distinguish MCDA [ 15]:

- Choice (optimization) methods,

- sorting methods,

- Ranking methods.

The outranking approach consists first in building, on the set of actions, a relation (called outranking relation) to represent the preferences of the decision maker. This relation is neither necessarily transitive nor complete ("incomparability" is a key outranking concept). The second step is the exploitation of this relation in order to help the decision maker in the choice, or sorting, or ordering problems [14]. Besides, [37] have described the outranking approach not to be bound into a mathematical model but providing further exploitation and processes to support the decision maker to conclude to a "good" decision.

The Preference Ranking Organization Method for Enrichment Evaluations (PROMETHEE) is based on a principle of pairwise comparisons [10]; [13]; [27]. PROMETHEE has the following principles [26]: evaluation table, relative importance, and information on decision maker preference.

\subsubsection{Evaluation table}

We consider a problem with a set of possible actions $A=\{a$, $b, \ldots\}$ which are evaluated on a set of $k$ criteria $K=\{k 1$, $k 2, . ., k j\}$. We can suppose, without loss of generality that all the criteria have to be maximized.

In the evaluation table provide by PROMETHEE, alternatives are evaluated on the different decision criteria, and these evaluations involve essentially numerical data.

\subsubsection{Information on decision maker preference}

For each criterion a specific preference function must be defined and there are corresponding parameters and thresholds. This function is used to compute the degree of preference associated to the best action in case of pairwise comparisons. Then, six possible shapes of preference functions are available and described in [27].

The decision-maker expresses his preference of action $a$ over action $b$ considering the criterion $\mathrm{kj}$ by computing a singlecriterion preference degree $\operatorname{Pj}(a, b)$ which is in function of $\operatorname{dj}(a, b)=g j(a)-g j(b)$. The value of this preference function $P j(a, b)$ is included between 0 and 1 , with:

- $\quad \mathrm{P}_{\mathrm{j}}(\mathrm{a}, \mathrm{b}) \approx \mathrm{o}$ if action $\mathrm{a}$ is not strictly preferred to action $b$;

- $\quad \mathrm{P}_{\mathrm{j}}(\mathrm{a}, \mathrm{b}) \approx 1$ if action $\mathrm{a}$ is not highly preferred to action $b$;

Several typical shapes are proposed [27] for the preference functions like the linear, the step or Gaussian preference function (see figure 1 for the function shapes).
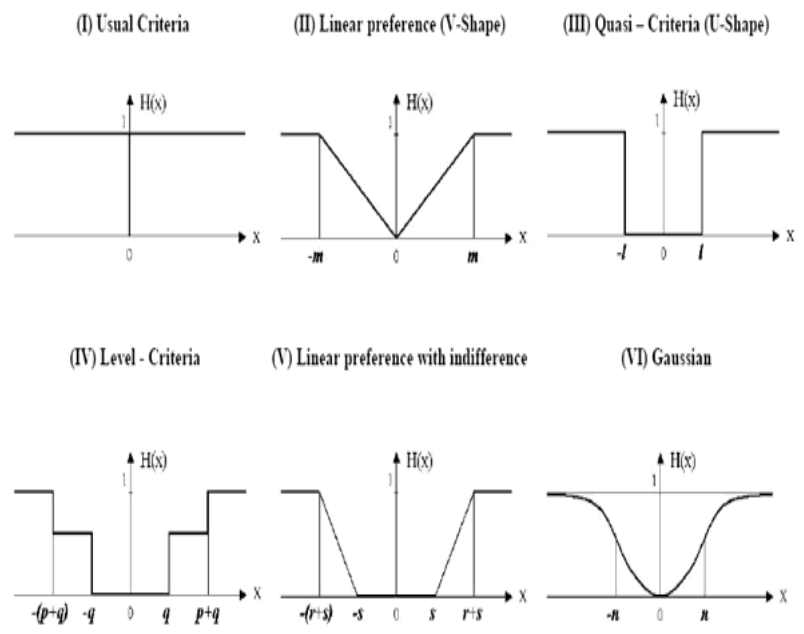

Fig 1. PROMETHEE preference functions. 


\subsubsection{Relative importance}

These pair-wise comparisons are aggregated to the positive and negative flows by using the weights $\mathrm{Wj}$ defined by the decision-maker.

PROMETHEE does not provided specific guidelines for determining these weights, but assumes that the decision maker is able to weigh the criteria appropriately, at least when the number of criteria is not too large [26]. Decision problem are required in a decision matrix, including $\boldsymbol{m}$ evaluation criteria, $\boldsymbol{n}$ alternatives and $\mathbf{n x m}$ evaluation.

PROMETHEE permits the computation of the following quantities for each criterion $\mathrm{j}(\mathrm{j}=1 . ., \mathrm{k})$ and alternatives $a$ and $b: P_{j}(a, b), \Phi_{j}+(a), \Phi_{j}-(a)$ and $\Phi_{j}(a)$.

For each alternative $a$, belonging to the set $A$ of alternatives, $P_{j}(a, b)$ is an overall preference index of $a$ over $b$, taking into account all the criteria, $\Phi j+(a)$ and $\Phi_{j}^{-}(a)$. These measure respectively positive and negative preference flows for each alternative. The positive flow is expressing how much an alternative is dominating (power) the other ones, and the negative flow how much it is dominated (weakness) by the other ones. $\Phi j(a)$ represents a value function, whereby a higher value reflects a higher attractiveness of alternative $a$. $\Phi j(a)$ is the net flow of alternative $a$ for criteria $k$. Then, based on these flows, the PROMETHEE I partial ranking is obtained.

The PROMETHEE I partial ranking provides a ranking of alternatives. In some cases, this ranking may be incomplete. This means that some alternatives cannot be compared and, therefore, cannot be included in a complete ranking. This occurs when the first alternative obtains high scores on particular criteria for which the second alternative obtains low scores and the opposite occurs for other criteria. The use of PROMETHEE I then suggests that the decision maker should engage in additional evaluation efforts [26].

PROMETHEE II provides a complete ranking of the alternatives from the best to the worst one. It is based on the balance of the two preference flows, and the net flow is used to rank the alternatives. The information looks stronger but some parts of it get lost in the process. Obviously the PROMETHEE I and II rankings are influenced by the weights allocated to the criteria [26].

\section{Positive flow:}

$$
\phi^{+}(a)=1 /(|A|-1) \sum_{x \in A} \sum_{j} \operatorname{WjPj}(a, x) ; \quad j=1, \ldots, k .
$$

Where:

$|\mathrm{A}|$ : cardinal of the set of actions $A$;

$w j$ : weight of criteria $\mathrm{kj}$;

$\operatorname{Pj}(a, x)$ : preference of action $a$ over $x$ on criterion $k j$.

$K$ : number of criteria

This score represents the global strength of action $a$ in comparison to all the other actions. Indeed, this score has to be minimized.

\section{Negative flow:}

$$
\phi(a)=1 /(|A|-1) \sum_{x \in A} \sum_{j} \operatorname{WjPj}(x, a) ; \quad j=1, \ldots, k .
$$

This score represents the global weakness of $a$ in comparison to all the other actions. Indeed, this score has to be minimized.
However, the two flows are usually combined to obtain the net flows defined as follows:

$$
\phi(a)=\phi^{+}(a)-\phi(a)
$$

This leads to the complete PROMETHEE II pre-order:

- $a$ is preferred to $b$ if $\phi(a)>\phi(b)$.

- $a$ is indifferent to $b$ if $\phi(a)=\phi(b)$.

Let us remark that the net flow can also be written as [Mareschal et al., 2008]:

$$
\phi(a)=1 /(|A|-1) \sum W_{j} \phi_{j}(a) \quad j=1, \ldots, k
$$

Where: $\quad \phi_{j}(a)=\sum\left(P_{j}(a, x)-P_{j}(x, a)\right) / x \in A$ is the single criterion net flow of criterion $\mathrm{Kj}$.

\subsection{Interacting criteria and Choquet integral}

All the multicriteria methods use the weighted arithmetical mean to incorporate information characterizing the preferences of the decision maker on the whole of the criteria [31], this supposes that the criteria are preferentially independent. However, in reality, the criteria interact and the assumption of preferential independence is seldom checked.

Reference [31] gives an overview of different types of interaction among criteria that could exist in the decision making problem. Three kinds of interaction defined and described in [31] are as follows: correlation, complementary, and preferential dependency. Clearly, when such complex interactions exist among criteria, it is necessary to use a welldefined weighting function on a subset of criteria rather than a single criterion during global evaluation. One such methodology for evaluation is the Choquet integral [1].

Thus, the integral of Choquet aims to improve the power of the multicriteria analysis by generalizing the weighted arithmetical mean by the taking into account of the interaction between the criteria. It also makes it possible to model the phenomena of interaction between criteria such as the correlation and the preferential dependence [25].

In order to define the integral of Choquet formally, we introduce as a preliminary the concept of capacity, on which the integral of Choquet is based.

Definition 1 [1] . Capacity $(\mu)$ : let $\mathrm{N}=\{1,2, \ldots, \mathrm{N}\}$ a set of criteria. A capacity on $\mathrm{N}$ is a function $\mu$ :

$2^{\mathrm{N}} \rightarrow[0,1]$ checking: $\mu(\varnothing)=0, \mu(\mathrm{N})=1$ and $\mu(\mathrm{A}) \leq \mu(\mathrm{B})$ if $\mathrm{A}$ is included in $\mathrm{B}$.

Definition 2 [1] .Choquet Integral: Let $\mu$ is a capacity on $\mathrm{N}$ and $\mathrm{F}: \mathrm{N} \rightarrow \mathrm{R}$ a function representing the scores of an alternative on $\mathrm{N}$ criteria. The Choquet integral of $\mathrm{F}$ compared to $\mu$ is given by:

$$
C_{\mu}(f)=\sum[f(\sigma(i))-f(\sigma(i-1))] \mu\left(A_{i}\right) ; i=1, \ldots, n
$$

with $A_{i}=\{\sigma(i), \sigma(i+1), \ldots, \sigma(n)\}, f(\sigma(0))=0$ and $\sigma$ is $a$ permutation in $\mathrm{N}$ such as $f(\sigma(1)) \leq f(\sigma(2)) \leq \ldots \leq f(\sigma(n))$.

Thanks to the use of a capacity, the integral of Choquet makes it possible to express preferences more sophisticated than the balanced sum.

Definition 3 [36]. fuzzy measurement:

A fuzzy measurement of a set $\mathrm{X}$ is a function $\mathrm{g}: 2^{\mathrm{X}} \rightarrow[0,1]$ such as the following conditions are satisfied: 
(1) $g(\emptyset)=0$;

(2) if $A, B \in F$ and $A \subseteq B$ then $g(A) \leq g(B)$;

(3) $g(X)=1$;

Or $g$ (A) indicates the weight of importance for a set A. the fuzzy measurement of $\mathrm{A}$ is called additive if: $g(A \cup B)$ $=g(A)+g(B)$ with $A \cap B=\phi$;

Supper additive if: $g(A \cup B) \geq g(A)+g(B)$ with $A \cap B=\phi$;

And sub additive if: $g(A \cup B) \leq g(A)+g(B)$ with $A \cap B=\phi$.

Definiton 4 [36]. Either $\mathrm{X}=\{\mathrm{x} 1, \mathrm{x} 2, \mathrm{x} 3, \ldots \ldots . . \mathrm{xn}\}$ a finished set and considers the $\lambda \in(-1, \infty)$, a $\lambda$ - fuzzy measurement is a function $\mathrm{g}_{\lambda}: 2^{\mathrm{X}} \rightarrow[0.1]$ such it meets the following conditions:

1. $g_{\lambda}(X)=1$;

2. If $A, B \in 2^{X}$ then, $g_{\lambda}(A U B)=g_{\lambda}(A)+g_{\lambda}(B)+\lambda g_{\lambda}(A)$ $\mathrm{g}_{\lambda}(\mathrm{B})$ with: $\mathrm{A} \cap \mathrm{B}=\phi$.

$\lambda$-fuzzy measurement can be formulated like [Leszczynski and $\mathrm{Al}, 1985]$ :

$$
\lambda+1=\Pi_{i}\left(\lambda g_{i}+1\right), i=1, \ldots, n .
$$

The value of $\lambda$ has three cases, as follows: $i=1, \ldots, n$

$$
\begin{aligned}
& \text { 1. if } \sum_{i} g_{i}>g(X) \text { then }:-1<\lambda<0 . \\
& \text { 2. if } \sum_{i} g_{i}=g(X) \text { then }: \lambda=0 . \\
& \text { 3. if } \sum_{i} g_{i}<g(X) \text { then }: \lambda>0 .
\end{aligned}
$$

\section{PROMCHOQ: A MCDA FOR ALTERNATIVES RANKING}

We consider a problem with a set of alternatives:

$\mathrm{A}=\{\mathrm{a}, \mathrm{b}, \mathrm{c}, \ldots\}$, which are evaluated on a set of $\mathrm{j}$ criteria $=$ $\left\{\mathrm{k}_{1}, \mathrm{k}_{2} ., \mathrm{k}_{\mathrm{j}}\right\}$.

In the table of evaluation, the alternatives are evaluated on the various decision criteria, and these evaluations imply primarily numerical data.

The Promchoq method is proposed and employed to obtain the full ranking of the alternatives (actions). As depicted in Figure 2, PromChoq is composed by four (4) steps. Compared with Promethee method, step 1 and step 2 are the same just for the calculation of monocriterion flow we use the formula (monocrterion flow $\phi$ of the action $\mathrm{a}_{\mathrm{i}}$ by taking in account the criterion $\mathrm{k}$ ):

$$
\phi_{k}\left(a_{i}\right)=1 /(N-1) \sum\left\{\begin{array}{c}
\left.P_{k}\left(a_{i}, a_{j}\right)-P_{k}\left(a_{j}-a_{i}\right)\right\} ; \text { aje } \mathrm{A} ; \mathrm{N} \text { criteria } \\
\text { number. }
\end{array}\right.
$$

As deference with Promethee method, PromChoq uses in step 3 the Choquet integral as aggregation operator and $\lambda$-fuzzy measure technique, in order to add the possibility to take in account the dependence between the criteria. Step 3 and 4 are presented below.

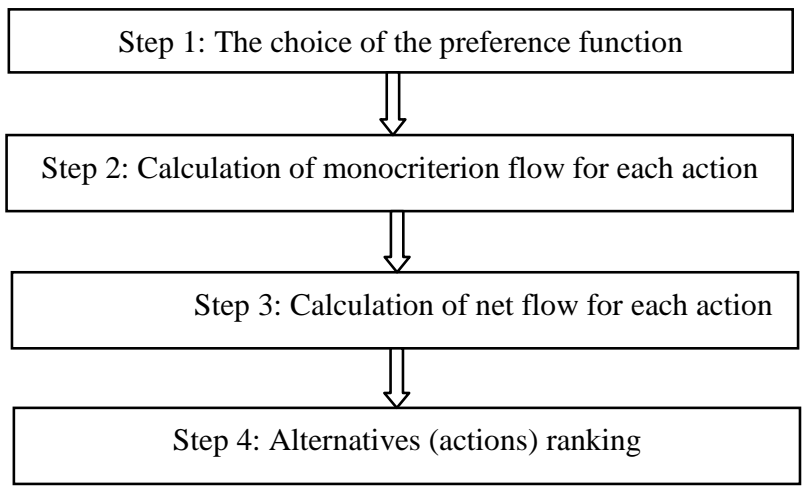

Fig 2. PromChoq steps.

Step 3. Calculation of net flow for each action

The objective of this step is to calculate net flow for each action (alternative), with PROMETHEE method, using the following balanced sum:

Net Flow: $\varnothing(a)=\sum w_{k} \emptyset_{k}(a) ; k=1, \ldots, N($ criteria $)$.

But as we explained in section 2.2, the balanced sum suffers from several limits, and thus, PROMETHEE method does not hold the interaction between the decision criteria. What poses constraints to use PROMETHEE method, for the problems of actions ranking, in the case, where the decision criteria interact between them.

To tackle that we propose to integrate the Choquet integral as operator of aggregation, because the Choquet integral was proposed to generalize additive measurements. This integral is based on the notion of the capacity like already explained in section 2.2. To do so, we use the technique $\lambda$-fuzzy measure, to calculate the capacity of each subset of criteria in order to take into account the interaction between the decision criteria's.

By using the equation of fuzzy measurement: $\lambda+1=\pi_{\mathrm{i}}\left(\lambda \mathrm{g}_{\mathrm{i}}+1\right)$ $\mathrm{i}=1, \ldots, \mathrm{n}$; we can calculate the value of $\lambda$. therefore the capacity for example of two criteria $(\mathrm{i}, \mathrm{j})$ which interact between them: $\mu(i, j)=\mu(i)+\mu(j)+\lambda \mu(i) \mu(j)$.

Then, by using the Choquet integral this capacity, we can calculate the Net flow of each action $\left(a_{i}\right)$ :

$$
C \mu\left(a_{i}\right)=\int \phi d \mu=\sum\left[\phi_{k}\left(a_{i}\right)-\phi_{k-1}\left(a_{i}\right)\right] \mu\left(A_{i}\right) .
$$

Or: $\mu\left(A_{i}\right)$ is the total score of the subset of criteria $\mathrm{A}_{\mathrm{i}}$.

In other words, we must calculate nets flows of each alternative with regard to all of the criteria: $C \mu\left(\mathrm{A}_{1}\right) ; C \mu\left(\mathrm{A}_{2}\right)$; $C \mu\left(\mathrm{A}_{3}\right) ; C \mu\left(\mathrm{A}_{4}\right) ; \ldots$

Step 4. Actions ranking

According to net flow of each action, there will be a descending order of flow what gives us a classification of the actions from the best one.

In order to help DM using PromChoq, we developed a tool which allows the automation of the competing actions classification (Figure 3). It was developed in JAVA under the NetBeans platform. 
The graphical interface contains seven interactive menus: with the launching of PromChoq, a principal window including the deferent functions of the application is posted. The decision maker has the possibility of filling the deferent forms, to seize the deferent criteria with their weights, and to choose the preference function of each criterion, and also s/he can seize the actions to be classified with its information.

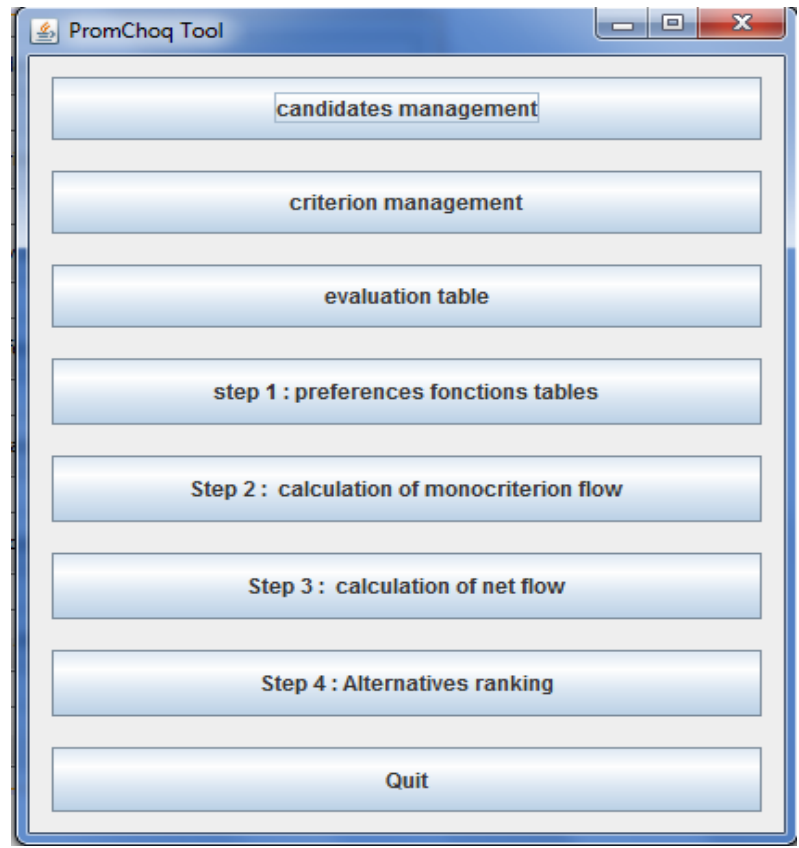

Fig 3. PromChoq tool- main menu

In the next section, we apply PromChoq to a real case study.

\section{CASE STUDY}

The previous section presented PromChoq and discussed advantages when using it. This section aims to operationalize it with a real-world system by using a case issued from Tebessa university (Algeria). The problem statement and the results of applying PromChoq are discussed below.

\subsection{Problem statement}

The present study was undertaken within the university of Tebessa in Algeria. The goal is classifying the candidates for the recruitment of teachers to the university by considering a set of well-defined criteria. Thus, it acts, according to the terminology adopted by [15], of arrangement problem (P $\gamma)$.

The process to solve this problem articulates around four principal steps:

1- Identifying the whole of the actions (candidates) to classify;

2- Drawing up a coherent list of classification criteria's;

3- Evaluating the performances of each action (candidate) according to the various criteria;

4- Applying the procedure of aggregation, to classify these candidates according to their total performances.

Firstly, we formulate the above problem by defining the set of actions, criteria, and the goal as follows.

Actions. $\Omega=\left\{a_{1}, a_{2}, \ldots a_{m}\right\}-$ set of candidates on which criteria is to be evaluated.
Criteria. In our case, the selection and the evaluation of the candidates in the recruitment of teachers, in higher education are based on the following five criteria:

$\underline{C}_{1}$ : the correspondence between the specialty of training of the candidate, and the requirements of the station required.

$\underline{\mathrm{C}}_{2}$ : the additional training of the diploma in the same field.

$\underline{\mathrm{C}}_{3}$ : work and studies carried out by the candidate in his field.

$\mathrm{C}_{4}$ : cumulated track records of the candidate.

$\underline{\mathrm{C}}_{5}:$ the result of the discussion with the jury of the selection.

Their weights are respectively $\mathrm{w}_{1}, \mathrm{w}_{2}, \mathrm{w}_{3}, \mathrm{w}_{4}$ and $\mathrm{w}_{5}$.

Goal. Within this case study, the candidates must be arranged by decreasing order of priorities in order to direct the efforts and to help the team of the administration in charge of the selection, to select the best candidates who satisfy the deferent classification criteria's.

\subsection{PromChoq application}

First, the criteria' weights are presented in the table below: we had the weights of the selection criteria after the study of the decree of the public function ministry which describes the recruitment conditions.

\section{Table 2. Criterion weight}

\begin{tabular}{|c|c|}
\hline Criterion & Weight \\
\hline $\mathrm{C}_{1}$ & 0,25 \\
\hline $\mathrm{C}_{2}$ & 0,25 \\
\hline $\mathrm{C}_{3}$ & 0,10 \\
\hline $\mathrm{C}_{4}$ & 0,20 \\
\hline $\mathrm{C}_{5}$ & 0,20 \\
\hline
\end{tabular}

For the candidates constituting the set of the actions, the scores were allotted in function to each of the five criteria. The results of these notations are gathered in the performances table. In our case, we have 5 candidates, who had the notes presented in Figure 4. Where each candidate is evaluated in function of each criterion from the selection criterions set. These two steps do not constitute steps of the method, but they present entry parameters for Promchoq method.

\section{Step 1. The choice of the preference function}

For our case we chose the function of the usual criterion Because, to classify the candidates, even a very small difference between the evaluations of two candidates: cand 1 and cand2, by taking in account the criterion $\mathrm{Cr}_{\mathrm{i}}$ (gj (cand1) gj (cand2)), it gives us a strict preference for the action having the evaluation most raised between the two candidates. 


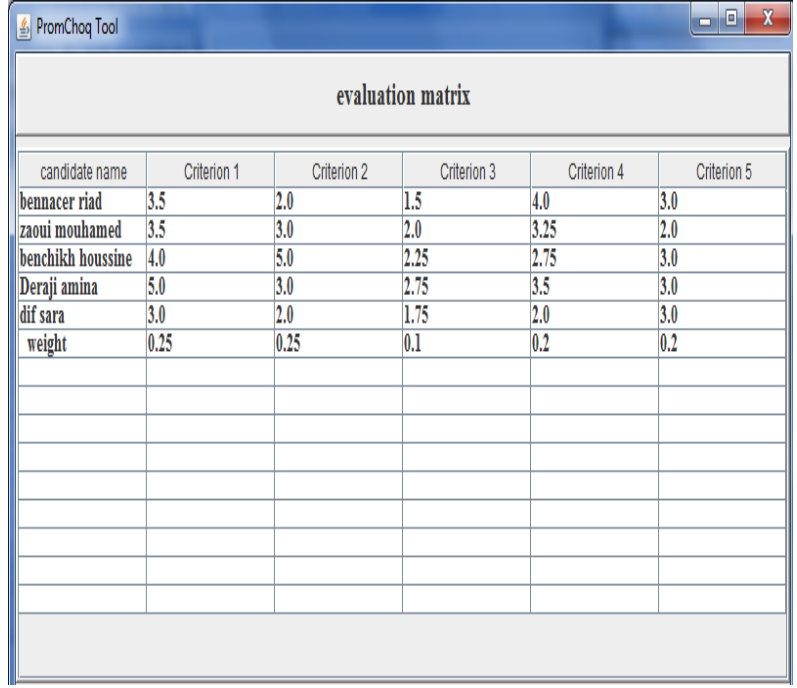

Fig 4: evaluation matrix

At the end of this step, we obtain a matrix of the preference function for each criterion. Figure 5 shows the obtained result for the first criterion.

\section{Example:}

According to the criterion $\mathrm{C}_{1}$ :

$\mathrm{P}_{\mathrm{C} 1}($ cand 1 , cand 2$)=\mathrm{g}_{\mathrm{C} 1}($ cand 1$)-\mathrm{g}_{\mathrm{C} 1}($ cand 2$)=3,5-3,5=0$ $\rightarrow \mathrm{P}_{\mathrm{c} 1}($ cand 1 , cand 2$)=0$;

The candidate cand 1 is not preferred to candidate cand2 according to $\mathrm{C}_{1}$.

$\mathrm{P}_{\mathrm{C} 1}($ cand 1 , cand5 $)=\mathrm{g}_{\mathrm{C} 1}($ cand 1$)-\mathrm{g}_{\mathrm{C} 1}($ cand5 $)=3-2=1 \rightarrow \mathrm{P}_{\mathrm{c} 1}$ (cand1, cand2) $=1$;

The candidate cand 1 is highly preferred to candidate cand5 according to $\mathrm{C}_{1}$.

\begin{tabular}{|c|c|c|c|c|c|}
\hline \multicolumn{5}{|l|}{ PremChog Tool } & $\therefore \quad 0 \quad x$ \\
\hline \multicolumn{6}{|c|}{ Step NN1: Matrix of preference functions } \\
\hline Criterion N ${ }^{\circ} 1$ & Criterion N² & Criterion N³ & 3 Criterion $\mathrm{N}^{\circ}$ & 4 Criterion $N^{\circ}$ & \\
\hline & Candidat N¹ & Candidat N² & Candidat $N^{\circ} 3$ & Candidat $N^{\circ} 4$ & Candidat No5 \\
\hline Candidat $\mathrm{N}^{0} \mathrm{l}$ & - & 0 & 0 & 0 & 1 \\
\hline Candidat $\mathrm{N}^{2}$ & & - & 0 & 0 & 1 \\
\hline Candidat $\mathrm{N}^{\circ} 3$ & & 1 & - & 0 & 1 \\
\hline Candidat $\mathrm{N}^{\circ} 4$ & & 1 & 1 & - & 1 \\
\hline Candidat $\mathrm{N}^{\circ 5} \mathrm{C}$ & & 0 & 0 & 0 & - \\
\hline
\end{tabular}

Fig 5: matrix of preference functions

\section{Step 2. Calculation of monocriterion flow}

In this step we calculated the monocriterion flow of our candidates with taking in account each criterion. The obtained results are presented in Figure 6.

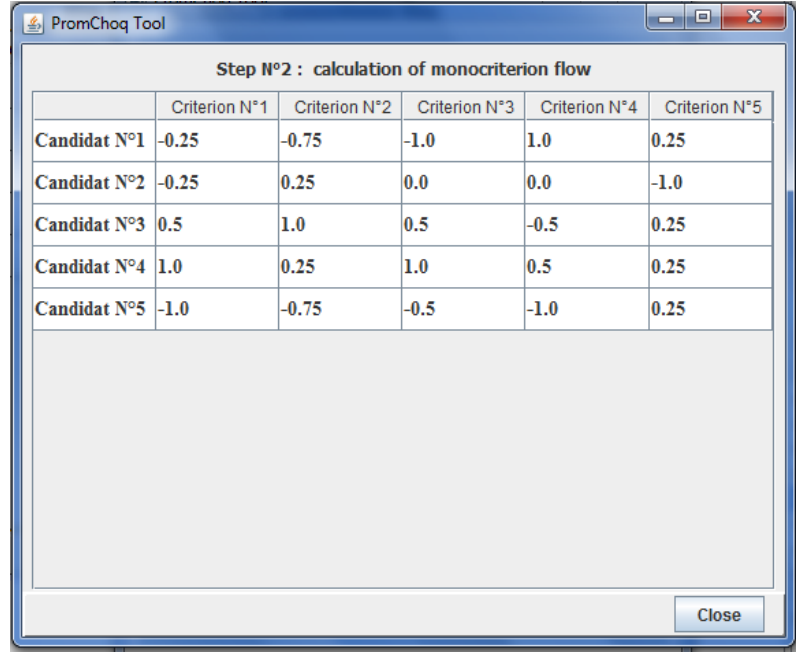

Fig 6: monocriteria flow

\section{Example:}

$\varnothing_{\mathrm{c} 1}(\mathrm{cand} 1)=1 / 4\left[\left[\mathrm{P}_{\mathrm{cl}}(\mathrm{cand} 1, \mathrm{cand} 2)-\right.\right.$

$\mathrm{P}_{\mathrm{cl}}(\mathrm{cand} 2$, cand 1$\left.)\right]+\left[\mathrm{P}_{\mathrm{cl}}(\right.$ cand1, cand 3$)-\mathrm{P}_{\mathrm{cl}}($ cand 3 , cand1 $\left.)\right]+$

$\left[\mathrm{P}_{\mathrm{cl}}(\right.$ cand 1 , cand 4$)-\mathrm{P}_{\mathrm{cl}}($ cans4, cand 1$\left.)\right]+\left[\mathrm{P}_{\mathrm{cl}}(\right.$ cand1, cand5) -

$\mathrm{P}_{\mathrm{c} 1}(\mathrm{cand}$ 5,cand1)]].

$\emptyset_{\mathrm{c} 1}($ cand 1$)=1 / 4[(0-0)+(0-1)+(0-1)+(1-0)]$.

$\emptyset_{\mathrm{c} 1}(\mathrm{E} 1)=-1 / 4=-0,25$.

This flow presents the weakness of the cand 1 with taking in account criteria $\mathrm{C}_{1}$, comparatively to the others candidates.

\section{Step 3. Calculation of net flow}

In this step we calculated the net flow of each candidate with taking in account the entire selection criterion. The results of calculations are presented at the figure blow.

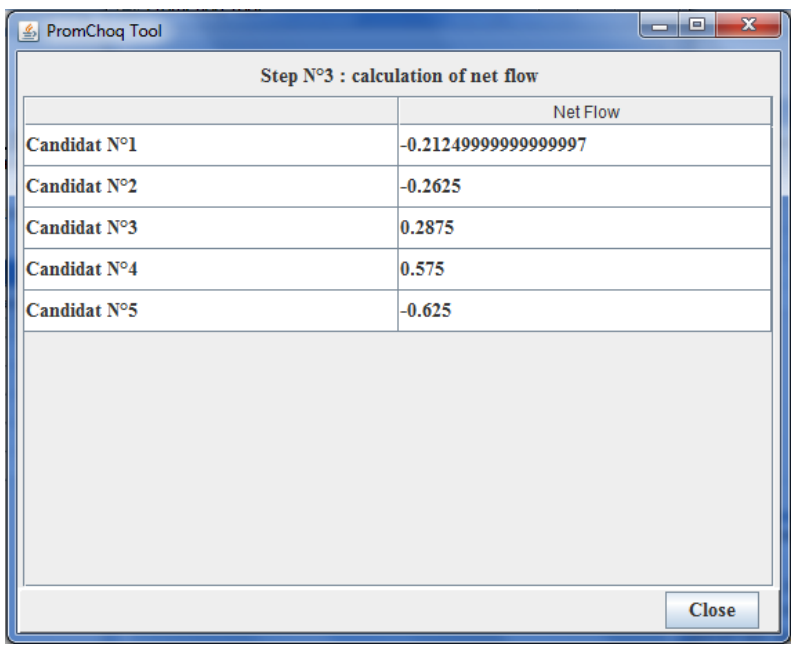

Fig 7: net flow

Example:

$\phi_{\text {cand } 1}=C \mu($ cand 1$)=\sum \varnothing_{k}($ cand 1$)-\emptyset_{k-1}($ cand 1$) \mu\left(A_{i}\right)$.

$\phi_{\text {cand1 }}=\left[\phi_{1}(\right.$ cand 1$)-\phi_{0}($ cand 1$\left.)\right] * \mu\{\operatorname{cr} 1, \mathrm{cr} 2, \operatorname{cr} 3, \operatorname{cr} 4, \operatorname{cr} 5\}+$ $\left[\phi_{2}(\right.$ cand 1$)-\phi_{1}($ cand 1$\left.)\right] * \mu\{\operatorname{cr} 2, \operatorname{cr} 3, \operatorname{cr} 4, \operatorname{cr} 5\}+\left[\phi_{3}(\right.$ cand 1$)-$ $\left.\phi_{2}(\mathrm{cand} 1)\right] * \mu\{\mathrm{cr} 3, \mathrm{cr} 4, \mathrm{cr} 5\}+\left[\phi_{4}(\mathrm{cand} 1)-\phi_{3}(\mathrm{cand} 1)\right]$ $* \mu\{\mathrm{cr} 4, \mathrm{cr} 5\}+\left[\phi_{5}(\mathrm{cand} 1)-\phi_{4}(\mathrm{cand} 1)\right] * \mu\{\mathrm{cr} 5\}$. 
$\phi_{\text {cand } 1}=-1 * 1+(-0,75+1) * 0,75+(0,25+0,75) * 0,5+$

$(0,25+0,25) * 0,4+(1-0,25) * 0,2$.

$\phi_{\text {cand } 1}=-1+0,1875+0,25+0,2+0,15$.

$\phi_{\text {cand } 1}=-0,2125$.

This net flow presents the weakness of the cand1 with taking in account of all the selection criteria, comparatively to the others candidates.

\section{Step 4. Alternatives ranking}

After obtaining the net flow of each candidate, we arranged these values in descending order, to obtain the candidate arrangement.

The result is presented below in Figure 8 .

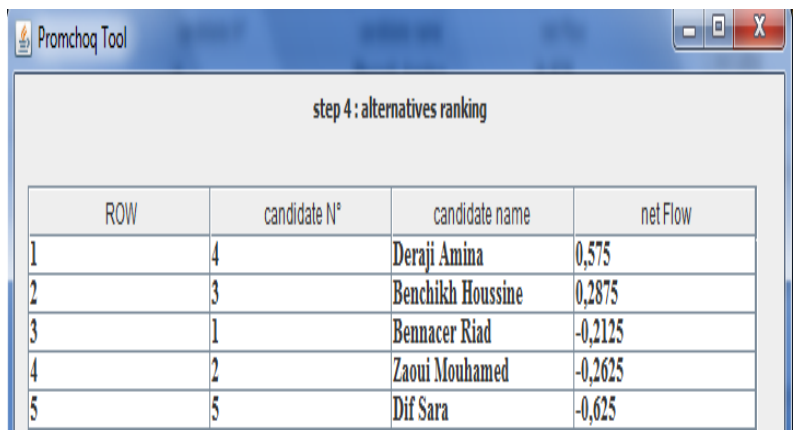

Fig 8: Candidates ranking

In addition to the final arrangement, results of an analysis of sensitivity to the variation of the weights allotted to the criteria. The table gives the intervals inside which we can vary the weight associated with the criterion considered, without the final classification not being affected (it should be noted that this variation relates to only one criterion at the same time). This reveals that the final classification is nevertheless rather stable.

\begin{tabular}{|c|c|}
\hline Criterion & Interval \\
\hline Criterion 1 & {$[0,01-0,99]$} \\
\hline Criterion 2 & {$[0,01-0,99]$} \\
\hline Criterion 3 & {$[0,01-0,2]$} \\
\hline Criterion 4 & {$[0,01-0,99]$} \\
\hline Criterion 5 & {$[0,01-0,99]$} \\
\hline
\end{tabular}

\subsection{Discussion and comparison}

In the previous section, we studied a practical case of application having for goal to use the multicriterion method PromChoq within the real data. We had the evaluations of five candidates out of five criteria determined by the administration of the university.

The developed method produces good performances in term to order the candidates, by the calculation of Net flow of each candidate, by taking into account all the criteria and their interactions, the classification is done by arranging flows in a decreasing order, This facilitates the interpretation of the results by the user.
In conclusion, these results show that the developed procedure can be applied successfully in the classification's problem in the multicriterion decision-aid field.

To show the effectiveness of Promcoq method we made a comparison study between it and PROMETHEE method (using an associated tool called PROMCALC for the same input data). The obtained results indicate that the improved method is more precise. Thus, each action is positioned compared to its flow Net.

\section{CONCLUSION AND PERSPECTIVES}

The decision-making aid is a very wide and complex field. To help a decision maker to choose the best solution in a new field is a difficult task. In this paper, the objective of our work was to set up a multicriterion method of analysis of decisionmaking aid, to solve the classification problems. To achieve this goal, we treated the methods known in the field of decision-making aid. Then, we studied PROMETHEE method considered as being adopted more for the classification. However, we noted that it has limits in the real situations, as regards taking into account the interaction of the criteria. PromChoq, the method presented in this paper may be considered as an extension to Promethee method to overcome this limit. To do so, we used $\lambda$ - fuzzy measure to evaluate weights of each sub-set of criteria and Choquet integral as an aggregation operator to obtain a global score for each alternative. To help decision maker to use our method, we implemented it in the NetBeans, which is portable and open source, to give a robust and effective tool to the user to classify alternatives.To show the utilisability and the effectiveness of PromChoq, our suggested method, we applied it and then Promethee method to a real case of classification of teachers candidate in a recruitment competition. After comparison, the obtained result was considered as very promising.

Our future work includes further improvement of PromChoq method and applying it to further real cases. Two possible areas for exploration, for example, would be the integration of fuzzy measurement, i.e. taking in account of the situations or the decision maker is hesitant and he has not a clear preference and the combination between this method and AHP (Analytic Hierachy Process) method [7], to profit from its advantage of the hierarchisation of the decision levels.

\section{ACKNOWLEDGEMENT}

The authors would like to deeply thank reviewers for their valuable comments and suggestions and the university of Tebessa which facilitated our case study

\section{REFERENCES}

[1] G. Choquet, Theory of capacities, Annales de l'Institut Fourier 5, 131-295. 1953.

[2] L.S. Shapley, A value for $n$-person games. In: H.W. Kuhn and A.W. Tucker (eds.), Contributions to the Theory of Games, Vol. II, Annals of Mathematics Studies, 28, (Princeton University Press, Princeton, NJ,), 307-317. 1953

[3] Von Neumann- Morgenstern, theory of' Gcrrnes and Econornic Behavior. Princeton University Press, Princeton. NJ. 1964.

[4] Benayoun et al, Linear Programming with Multiple Objective Functions: Step Method (STEM) , math. Programming. 366-375.1971. 
[5] Zionts-Wallenius, wallenius. AUD J. WALLEKIU, An Interactive Programing Method for Solving the Multiple Criteria Problem. management Sci.. 22 652-663. 1976.

[6] Keeney et Raïffa,D écisions with multiple objectives: preference and value tradeoffs, Series in probability and mathématical statistics, New York, 1976.

[7] Saaty, the Analytic hierarchy Process, McGrau-Hill, New York. 1980.

[8] B. Roy, 1985, Méthodologie multicritère d'aide à la décision, 1er Ed, Economica, Paris, 1985

[9] Alain Schärlig, Décider sur plusieurs critères: Panorama de l'aide à la décision multicritère, 1er Ed, Presse polytechniques romandes, Suisse, 1985.

[10] Brans, J. P. and Vincke, P. H. "A preference ranking organization method: The PROMETHEE method for multiple criteria decision-making", Management Science, 31, 647-656.

[11] Jacquet-Lagrèze, Meziani et Slowinski, "Assessing a Set of Additive Utility Functions for Multicriteria Decision-Making. The UT4 Method," Eiiroiiearl J.Oper. Res.. 10.151- 164. 1982.

[12] M. R. Mahmoud and L. Garcia, Comparison of different multicriteria evaluation methods of the Red Bluff Diversion Dam, Environmental Modeling and Software 15(5) (2000) 471-478.

[13] VINCKE, P. "L'aide multicritère à la décision". Bruxelles : Editions de l'Université de Bruxelles, 179 p. 1989.

[14] B. Roy, 1992: Cahiers du Lamsade $n^{\circ} 97$, Univ. Paris-Dauphine, 1992.

[15] B.ROY, 1993b: B.Roy, "Aide multicritère à la décision: methodes et cas", Economica, Paris, 1993.

[16] Murofushi, T. and Sugeno, M. "A Theory of Fuzzy Measures Representations, the Choquet Integral and Unll Sets", Journal of Mathematical Analysis and Application Vol.159, No.2,pp.532-549.2005.

[17] Saaty, T.L., "Theory and Applications of the Analytic Network Process", RWS Publications, Pittsburgh, USA, ISBN: 1-888603-06-2, 2005.

[18] Alain Schärlig, "Pratiquer Electre et Prométhée: un complément à décider sur plusieurs critères", Presses polytechniques et universitaires romandes, 1er Ed, Paris, 1996

[19] A. Albadvi, S. K. Chaharsooghi and A. Esfahanipour, Decision making in stock trading: An application of PROMETHEE, European J. Operational Research 177(2) (2007) 673-683.

[20] J.-L. Marichal, « Agregation operators for multicreteria decision aid », Thèse de doctorat. Univ. De Liege.258p, 1999.

[21] S.Ben Mena, «Introduction aux méthodes multicritères », Biotechnol Agron.Soc.Environ, 2000.

[22] Takahagi, Eiichiro: "On Identification methods of $\lambda$ fuzzy measures using weights and $\lambda$ ", Japanese Journal of Fuzzy Sets and Systems, vol.12, no.5, 665- 676, 2000
[23] Kaynak, T, Human Resources Management, Nobel Yayınevi, İstanbul. 2002.

[24] D. Boyssou, D. Dubois et all, " Concepts et Méthodes pour l'Aide à Décision », Hermès, 2003.

[25] J.-L. Marichal, « Fuzzy measures and integrals in the MCDA sorting problematic »,Thèse de doctorat.Univ. Libre de Bruxelles. 202p, 2003.

[26] Macharis C, Springael J, De Brucker K, Verbeke A (2004) PROMETHEE and AHP: The design of operational synergies in multicriteria analysis. Strengthening PROMETHEE with ideas of AHP. Eur J Oper Res 153:307-317.

[27] Brans J-P, Mareschal B," PROMETHEE methods. In Multiple Criteria Decision Analysis - State of the Art Surveys, Figueira J, Greco S, Ehrgott M (eds). Springer:New York; 163 -195,2005.

[28] Bouyssou , "Approches descriptives et constructives d'aide à la décision: Fondements et comparaison", Thèse de doctorat, Université Paris Dauphine, 2006.

[29] Chung, S. H., Lee, A. H. L., and Pearn, W. L., "Analytic Network Process (ANP) Approach for Product Mix Planning in Semiconductor Fabricator", International Journal of Production Economics, 96, 15-36. 2006.

[30] Elisabeth Rakus-Andersson, Claes Jogreus: "TheChoquet and Sugeno Integrals as Measuresof Total Effectiveness of Medicines. In: Theoretical Advances and Applications of FuzzyLogic and Soft Computing (Proceedings of IFSA 2007, Cancun, Mexico), eds: Oscar Castillo, Patricia Melin, Oscar Montiel Ross, Roberto Sepulveda Cruz, Witold Pedrycz, Janusz Kacprzyk, Springer-Verlag, Soft Computing 42, pp. 253-262.2007.

[31] Marichal, J.-L.: "An axiomatic approach of the discrete Choquet integral as a tool to aggregate interacting criteria" IEEE Trans.Fuzzy Systems, vol. 8, pp. 800-807, Dec. 2000.

[32] Sridhar P., M. Madni A., and Jamshidi M., "Multicriteria decision making in sensor network", IEEE Instrumentation \& Measurement Magazine, 11(1), PP-24-29, 2008.

[33] Fodor, J. \& Roubens, M. (1994). Fuzzy Preference Modeling and Multicriteria Decision Support. Kluwer, Dordrecht.

[34] Harris R ,1998 ,Introduction to decision making.

[35] Lu J, Zhang G, Ruan D, Wu F (2007) Multiobjective group decision making: methods, software and applications with fuzzy set techniques. Imperial College, London.

[36] M.SUGENO, Theory of fuzzy integrals and its applications, $\mathrm{PhD}$ thesis, Tokyo Institute of Technology, 1974.

[37] Siskos, J., Spyridakos, A. et D. Yannacopoulos, 1993,"MINORA: A Multicriteria Decision Aiding System for Discrete Alternatives", Journal of Information Science and Technology 2, 2, 136-149. 